\title{
Differential invading potential among cryptic species of a Lessepsian bioinvader, the blotchfin dragonet Callionymus filamentosus
}

\author{
Daniel Golani ${ }^{1}$, Giacomo Bernardi ${ }^{2, *}$ \\ ${ }^{1}$ Department of Evolution, Systematics and Ecology, The Hebrew University of Jerusalem, 91904 Jerusalem, Israel \\ ${ }^{2}$ Department of Ecology and Evolutionary Biology, University of California Santa Cruz, 100 Shaffer Road, Santa Cruz, \\ California 95060, USA
}

\begin{abstract}
The bioinvasion of the Mediterranean from the Red Sea via the Suez Canal, called Lessepsian bioinvasion, was used as a way to uncover the invading potential of different genetic clades. Populations of blotchfin dragonet Callionymus filamentosus were assessed, based on 2 mitochondrial markers (control region and cytochrome oxidase 1) and 2 nuclear markers (2nd intron of the ribosomal protein S7 and rhodopsin). Populations clustered in 2 mitochondrial clades: one clade included only individuals collected in the Red Sea, while the other included samples from both the Red Sea and the Mediterranean. In contrast, nuclear markers did not separate Red Sea and Mediterranean individuals. We interpret these results as a differential in invading potential of the individuals belonging to the 2 mitochondrial clades. Incomplete lineage sorting in nuclear markers suggests that these 2 groups are at an early stage of differentiation. Thus, the model of Lessepsian bioinvasions may, in some cases, be used to assess ecological differences among non-geographic clades.
\end{abstract}

KEY WORDS: Cryptic species · Callionymus filamentosus $\cdot$ Biological invasion $\cdot$ Lessepsian migration $\cdot$ Mediterranean Sea

\section{INTRODUCTION}

The increasing use of genetic markers in ecological and evolutionary studies has revealed a number of surprises including the evidence of significant numbers of species that are morphologically identical but genetically different, the so-called cryptic species (Knowlton 2000). Cryptic species are defined as genetically distinct entities that are morphologically indistinguishable. In a study on unicornfishes, Horne et al. (2008) found that different species showed no population structure across known biogeographic barriers (between the Indian Ocean and the Pacific), but displayed similar patterns of well-resolved sympatric and reciprocally monophyletic clades that were called non-geographic clades. They attributed these patterns to undefined, but likely historical and ecological, factors. Ultimately, these clades may diverge into cryptic species. The situation may reflect the early stages of speciation or a later stage of speciation where populations are not in an environment favoring morphological changes. The number of cryptic species is difficult to estimate, but it is likely to be considerable; it is also likely to be highest in environments that are difficult to fully assess, such as for marine species (Knowlton 2000). Identifying cryptic species is difficult and, while tallying cryptic species is essential to evaluate biodiversity, understanding their ecology is complex because by definition they can only be identified by genetic means. Therefore, very few studies have addressed the ecological significance of cryptic speciation, with some notable exceptions (Hebert et al. 2004, Smith et al. 2007). For such a study to be performed, one needs to be in a situation where cryptic species will have different ecological characteristics and can easily be identified. One potential example would be the case of biological invasions, where different species have different invading potential. 
The opening of the Suez Canal in 1869 resulted in a movement of marine organisms, termed Lessepsian migration (after the Canal engineer Ferdinand de Lesseps), and initiated a process of invasion from the Red Sea into the Mediterranean. To date, about 80 bony fishes are considered to be Lessepsian migrants (Golani 2010, Bariche \& Heemstra 2011). In general, it is predicted that invading populations are genetically depressed due to the small number of effective original migrants, a phenomenon known as founder effect, resulting in a genetic bottleneck (e.g. Azzurro et al. 2006). In contrast, all Lessepsian species, except for the recently recorded bluespotted cornetfish Fistularia commersonii, showed very high levels of genetic diversity and were essentially indistinguishable from their source (Red Sea) populations (Golani et al. 2007, Bernardi et al. 2010). Since the focal species were early Lessepsian migrants (first Mediterranean record between 1902 and 1964), one simple explanation was that the lack of a genetic bottleneck resulted from multiple invasions, which slowly built up the genetic diversity observed in Mediterranean populations (Bucciarelli et al. 2002, Hassan et al. 2003, Hassan \& Bonhomme 2005, Azzurro et al. 2006).

In the present study, we use the blotchfin dragonet (also known as filamentous dragonet) Callionymus filamentosus as our focal species. C. filamentosus is a small fish (max. size $20 \mathrm{~cm}$ TL) that lives on sandy and muddy bottoms and that is originally distributed over most of the Indo-Pacific from the Red Sea, Africa to Australia and Taiwan. It was first recorded in the Mediterranean along the Israeli coast, where it established a huge population (Ben-Tuvia 1953, Tortonese 1953). In subsequent years, it spread westward to the island of Rhodes (Pancucci-Papadopoulou et al. 2005).

Callionymus filamentosus is a benthic species, found in the Mediterranean at 20 to $60 \mathrm{~m}$ but occasionally in deeper waters to ca. $100 \mathrm{~m}$. This species manifests clear sexual dimorphism: only males have a filamentous first dorsal spine and the 2 median caudal rays are prolonged. It feeds upon small benthic invertebrates and has pelagic eggs and larvae (Golani et al. 2002). The pelagic larval duration (PLD) for this species is unknown, but the PLD of a species in the same family, Synchiropus splendens, was estimated to be 32 to $39 \mathrm{~d}$, a range that is fairly typical of coral reef species (Brothers \& Thresher 1985).

Our goal in the present study was to use Lessepsian bioinvasions to determine the differential invading potential of cryptic species. Using 2 mitochondrial and 2 nuclear markers, we looked at potential for cryptic speciation, and whether genetically diver- gent clades of Callionymus filamentosus presented different invading characteristics.

\section{MATERIALS AND METHODS}

\section{Collections and DNA samples}

Samples were collected by trawl and beach seine from 1995 to 2008 in the Red Sea and the Mediterranean, totaling 155 individuals, with 91 individuals from the Red Sea (Eilat, Israel) and 64 individuals from the Mediterranean (Jaffa, Israel). Entire specimens were preserved in $95 \%$ ethanol at room temperature, and DNA was extracted from muscle tissue following standard protocols (Sambrook et al. 1989).

\section{PCR amplification and sequencing}

Two mitochondrial markers (control region and cytochrome oxidase 1 [COI]) and 2 nuclear markers (2nd intron of the ribosomal protein S7 [S7_2] and rhodopsin [Rod]) were used in this study. All sequences were deposited in GenBank (Accession numbers JQ796921-JQ797305). Amplification of the 5 ' hypervariable portion of the mitochondrial control region (also called D-loop) used the universal fish primers CR-A and CR-E (Lee et al. 1995). The amplification of COI used fish specific primers VF2T1 and VR1dT1 (Ward et al. 2005). The amplification of the 2nd intron of the ribosomal protein S7 (S7_2) used the primers and protocols of Chow \& Hazama (1998). The PCR of the rhodopsin marker (Rod) followed the nested amplification protocols of Sevilla et al. (2007) with RHO30F and RHO 319R for the first set of primers and Rho F2x and RhoR4n for the second set of primers. After purification following the manufacturer's protocol (ABI, Perkin-Elmer), sequencing was performed with the primers used in the PCR amplification on an ABI 3100 automated sequencer (Applied Biosystems, Foster City).

\section{Phylogenetic analyses}

We used the computer program MAFFT (Katoh et al. 2002) implemented by the Geneious software package (Drummond et al. 2011) to align the DNA sequences. Phylogenetic relationships were assessed by maximum likelihood (ML, GARLI software, Zwickl 2006), maximum parsimony (MP, PAUP* ${ }^{*}$ software Swofford 2003), and neighbor-joining (NJ, 
PAUP software) methods. For maximum likelihood topologies, we conducted 10 independent runs in GARLI, using default settings and the automated stopping criterion, terminating the search when the ln score remained constant for 20000 consecutive generations. The best likelihood of those runs was retained and is presented here (Fig. 1). Maximum parsimony searches included 100 random addition replicates and TBR branch swapping with the Mul- trees option. Neighbor-joining reconstructions used distances based on substitution models obtained with Modeltest $(\mathrm{HKY}+\mathrm{G})$. Statistical confidence in nodes was evaluated using 2000 non-parametric bootstrap replicates (Felsenstein 1985) (100 replicates for maximum likelihood in GARLI, using the automated stopping criterion set at 10000 generations). Topological differences were tested using a Shimodaira and Hasegawa test (Shimodaira \& Hasegawa 1999)

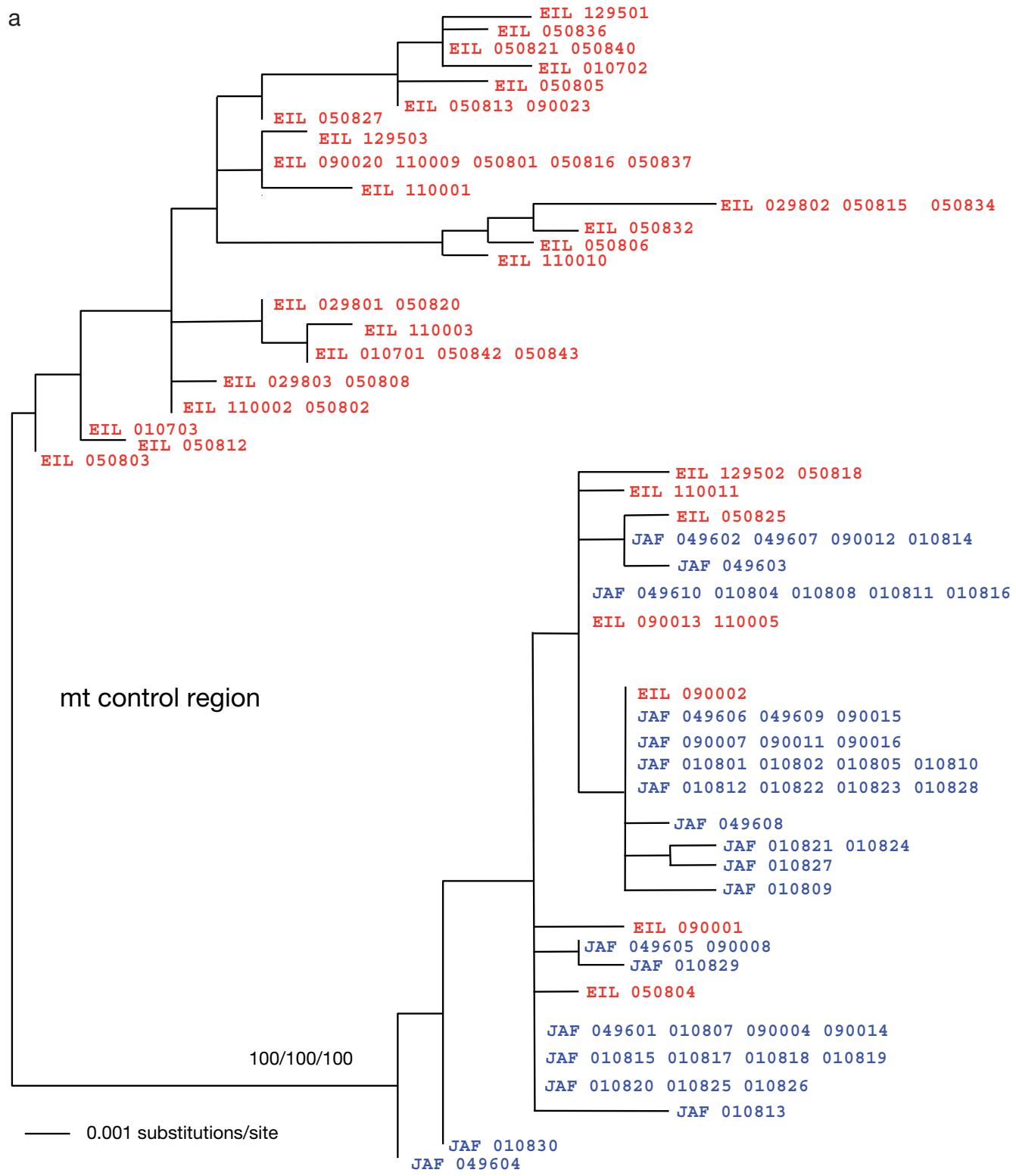

Fig. 1. (above and following pages) Callionymus filamentosus. Phylogenetic relationships of individuals based on (a) control region, (b) cytochrome oxidase 1 (COI), (c) 2nd intron of the ribosomal protein S7 (S7_2), and (d) rhodopsin (Rod) sequences, representing $(\mathrm{a}, \mathrm{b}) 2$ mitochondrial $(\mathrm{mt})$ and $(\mathrm{c}, \mathrm{d}) 2$ nuclear markers. Three reconstruction methods were used: maximum likelihood (shown here), neighbour-joining, and maximum parsimony, which resulted in identical topologies. Bootstrap support for the main clades $>50 \%$ for each of the methods used is shown next to the corresponding node. Individuals in red were collected in the Red Sea (Eilat, EIL); individuals in blue were collected in the Mediterranean (JAF and ISR). Sample numbers given in 6 digits correspond to month of collection, year of collection, and sample number (2 digits each) 
b

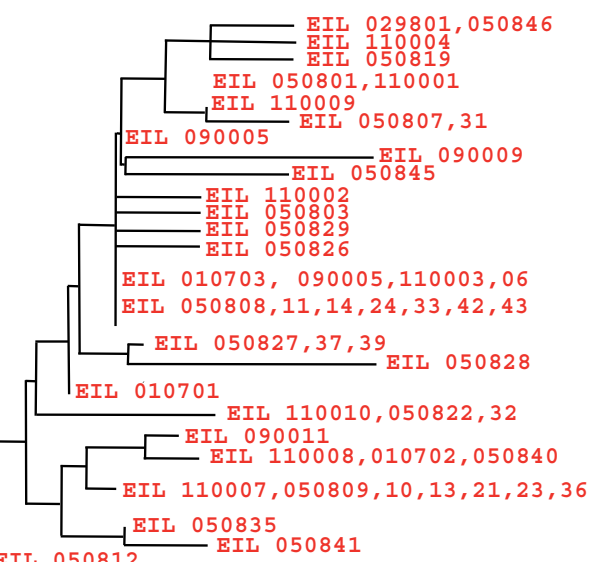

LEIL 029802,010704,050806,15,34

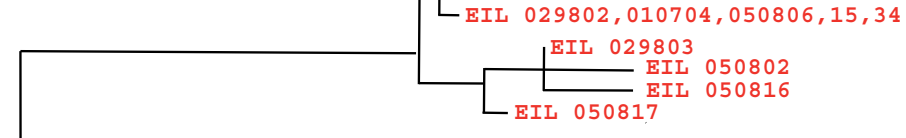

$\mathrm{mt} \mathrm{COI}$

EIL 090001 EIL $090002,050804,30,38$

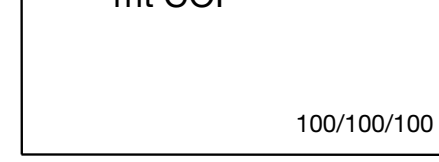

0.001 substitutions/site nuclear S7_2

C

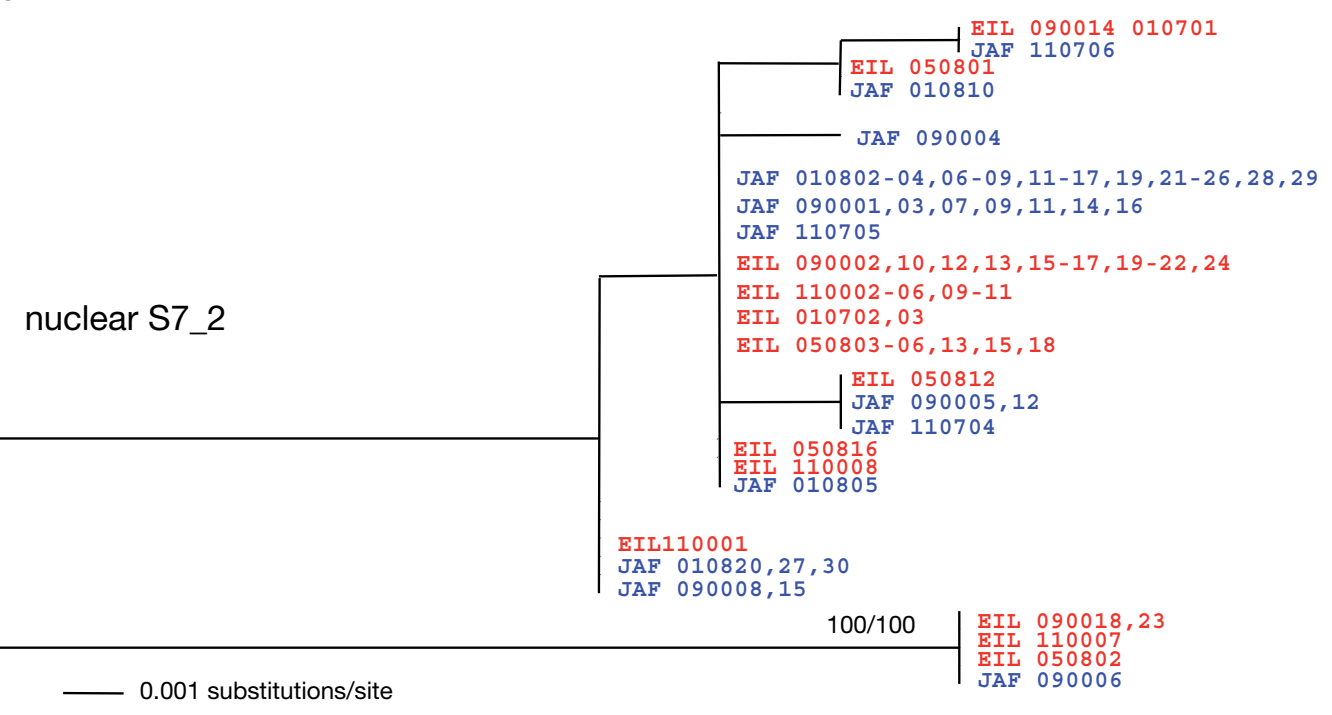

Fig. 1 (continued) 


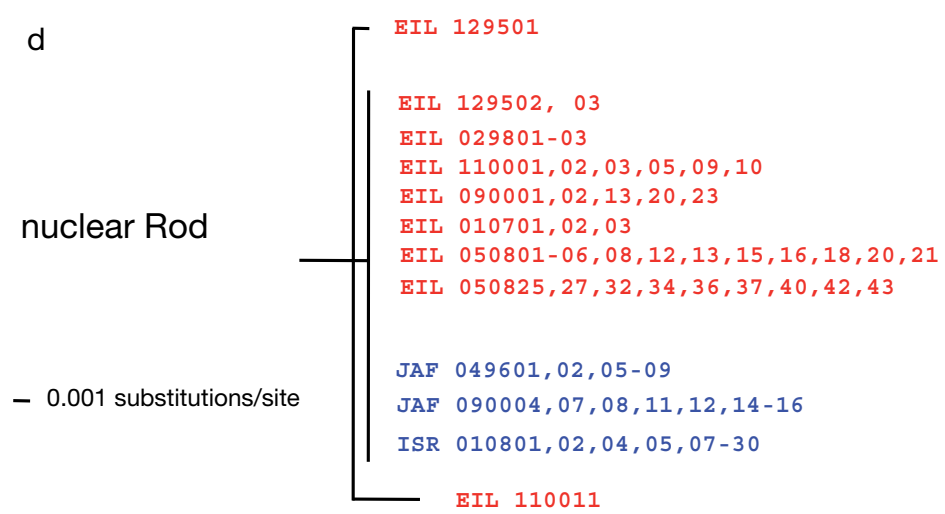

Fig. 1 (continued)

implemented by PAUP, based on resampling of estimated log-likelihoods tests (RELL, 1000 replicates). Genetic distances between clades were calculated using the pairwise distance of individuals between clades minus the paiwise distance of individuals within clades.

\section{Population analyses}

Number of haplotypes and haplotype diversity were calculated using the software package DNAsp (Librado \& Rozas 2009). Population structure (classical $F_{\mathrm{ST}}$ and $\left.\Phi_{\mathrm{st}}\right)$ and their associated statistical significance were calculated using Arlequin (Excoffier \& Lischer 2010) with 1000 random permutations.

\section{RESULTS}

\section{Sequences}

Some PCR reactions did not produce usable sequences, with older specimens yielding less sequences than newer specimens (2008), for which all samples worked. Thus, out of 155 sampled individuals, we obtained 90 control region sequences, 124 COI sequences, 84 S7_2 sequences, and 87 Rod sequences. Sequencing of the mitochondrial control region resulted in 368 aligned base pairs, with 33 variable and 26 phylogenetically informative positions. Sequencing of the mitochondrial COI resulted in 632 aligned base pairs, with 42 variable and 26 phylogenetically informative positions. The sequencing of the nuclear S7_2 fragment resulted in 457 aligned bases, with 16 variable and 15 informative positions, while the nuclear Rod marker resulted in 440 aligned bases and only 3 polymorphic sites.
None of the samples showed any heterozygous position at the nuclear loci, making the direct reading of the sequence straightforward and cloning unnecessary.

\section{Phylogenetic relationships}

The different phylogenetic methods yielded topologies that were not statistically significantly different (Shimodaira and Hasegawa test, $\mathrm{p}>0.4$ ), thus we decided to present results based on the Maximum Likelihood method (Fig. 1). Phylogenetic relationships based on mitochondrial markers resulted in 2 well-supported $(100 \%$ bootstrap replicates) reciprocally monophyletic assemblages (Fig. 1a,b). Most importantly, samples were not randomly distributed in the 2 clades. One clade (called Red Sea clade) included exclusively samples from the Red Sea, with 35 and 57 individuals, for the Dloop and COI markers, respectively. The other clade (called Lessepsian clade) included 9 and 11 samples collected from the Red Sea and 46 and 56 samples from the Mediterranean, from Dloop and COI, respectively.

Nuclear markers showed, as expected, lower levels of variability (Fig 1c,d). Rhodopsin sequences showed very little divergence; all individuals from the Red Sea and the Mediterranean were essentially identical. While 2 closely related species, Callionymus reticulatus and C. maculates, showed identical sequences (GenBank EU491962.1, EU491965, data not shown), the rhodopsin marker does have the power to identify some cryptic species (G. Bernardi unpubl.). So, the lack of resolution in C. filamentosus may not simply be due to lack of segregating power. Indeed, the 2nd intron of the ribosomal protein S7 showed variability, and shallow clades were recovered. All clades, however, included samples from both the Red Sea and the Mediterranean and did not match the separation found with the mitochondrial markers.

\section{Genetic distances}

Due to the low diversity obtained with nuclear markers, population level analyses were only performed on mitochondrial markers. Individuals from the 2 mitochondrial clades showed a high level of average pairwise genetic divergence, with $3.68 \%$ for Dloop and $2.45 \%$ for COI (Table 1). Pairwise genetic 
Table 1. Callionymus filamentosus. Mitochondrial markers characteristic of the study populations. Rows represent the 2 genetic markers investigated: control region (Dloop) and cytochrome oxidase 1 (COI). Columns provide information on the 2 mitochondrial clades (Red Sea and Lessepsian; \%: pairwise genetic divergence within clades) as well as on individuals, from the Red Sea and Mediterranean (Med.), within the Lessepsian clade. Pairwise sequence divergence between clades (btw) and a calculated divergence between clades (c. btw), computed as genetic distance (\%) between clades minus that within clades, are also provided. $\mathrm{n}$ : number of individuals; Hn: number of haplotypes; and Hd: haplotype diversity. Computation of pairwise sequence divergence within Red Sea and Mediterranean populations within the Lessepsian clade are not presented due to low sample numbers

\begin{tabular}{|c|c|c|c|c|c|c|c|c|c|c|c|c|c|c|c|c|}
\hline & \multirow{2}{*}{\multicolumn{4}{|c|}{ Red Sea clade }} & \multirow{2}{*}{\multicolumn{4}{|c|}{ Lessepsian clade }} & \multirow{3}{*}{$\begin{array}{c}\text { btw } \\
\%\end{array}$} & \multirow{3}{*}{$\begin{array}{c}\text { C. btw } \\
\%\end{array}$} & \multicolumn{6}{|c|}{ Lessepsian clade } \\
\hline & & & & & & & & & & & & Sea & nd. & & ed. in & \\
\hline & $\mathrm{n}$ & $\mathrm{Hn}$ & $\mathrm{Hd}$ & $\%$ & $\mathrm{n}$ & $\mathrm{Hn}$ & $\mathrm{Hd}$ & $\%$ & & & $\mathrm{n}$ & $\mathrm{Hn}$ & $\mathrm{Hd}$ & $\mathrm{n}$ & $\mathrm{Hn}$ & $\mathrm{Hd}$ \\
\hline DLoop & 35 & 22 & 0.96 & 1.56 & 55 & 19 & 0.87 & 0.59 & 3.68 & 1.51 & 9 & 7 & 0.94 & 46 & 14 & 0.84 \\
\hline COI & 57 & 27 & 0.94 & 0.39 & 67 & 14 & 0.83 & 0.24 & 2.45 & 1.81 & 11 & 4 & 0.78 & 56 & 11 & 0.77 \\
\hline
\end{tabular}

divergence within clades was lower for the Lessepsian clade $(0.59 \%$ for Dloop, $0.24 \%$ for COI) than for the Red Sea clade ( $1.56 \%$ for Dloop, $0.39 \%$ for COI). This resulted in a calculated genetic distance between clades of $1.51 \%$ for Dloop and $1.81 \%$ for COI (individuals pairwise divergence between clades minus individuals pairwise divergence within clades). The difference in genetic distance within clades also translated into a difference in number and diversity of haplotypes. Based on Dloop, the Red Sea clade comprised 35 individuals that partitioned into 22 haplotypes (Haplotype diversity, $\mathrm{Hd}=0.965$ ). The Lessepsian clade comprised 55 individuals that partitioned into 19 haplotypes ( $\mathrm{Hd}=0.87$ ). Based on COI, the Red Sea clade comprised 57 individuals that partitioned into 27 haplotypes (Haplotype diversity, $\mathrm{Hd}=0.937)$. The Lessepsian clade comprised 67 individuals that partitioned into 14 haplotypes $(\mathrm{Hd}=$ 0.83) (Table 1).

\section{Population structure}

While sampling was done using seine nets and fish were taken as a pool of sympatric individuals, genetic analyses revealed that in Eilat we collected more individuals that clustered with the Red Sea clade than with the Lessepsian clade. Indeed, 35 and 57 individuals clustered in the Red Sea clade while only 9 and 11 individuals clustered in the Lessepsian clade when using Dloop and COI, respectively (which corresponds to 22 and $16 \%$ of the collected individuals, respectively).

Within the Lessepsian clade, individuals from the Mediterranean exhibited a slight decrease in genetic diversity; haplotype diversity for Red Sea individuals was 0.94 and 0.78 for Dloop and COI, respectively, while it was 0.84 and 0.77 for samples collected in the Mediterranean (Table 1). Gene flow was evaluated within the Lessepsian clade between Red Sea and Mediterranean populations. When calculating $F_{\mathrm{ST}}$ using Dloop data, values were found be low (0.050) and non-significant $(\mathrm{p}=0.15)$. Data based on COI, however, showed higher $F_{\mathrm{ST}}$ values (0.43) that were significant $(p<0.0001)$. Our sample sizes were uneven, with 11 Red Sea individuals and 56 Mediterranean individuals. In order to determine whether sample size biased our results, we did 10 replicates where the 11 Red Sea individuals were compared to 11 Mediterranean individuals picked randomly out of the 56. All replicates showed the same pattern of high $F_{\mathrm{ST}}$ values (range $F_{\mathrm{ST}}=0.17-0.52$ ) that were statistically significant (range $\mathrm{p}=0.000-0.027$ ).

\section{DISCUSSION}

\section{Population structure of Lessepsian migrants}

The genetics of biological invasions often defy classical assumptions, and Lessepsian bioinvasions are no exception. In general, it is assumed that few individuals are effective invaders, resulting in a signature of genetic bottleneck (Geller et al. 2010). With very few exceptions, genetic bottlenecks were not found in Lessepsian bioinvaders (Bernardi et al. 2010). Callionymus filamentosus follows this trend, with high levels of gene flow between Red Sea and Mediterranean populations, and a very mild reduction of genetic diversity in the invading population. The reduced sample size of Red Sea individuals in the Lessepsian clade prevents definitive conclusions; yet, the presence of some population structure at the COI marker may be indicative of reduction in gene flow across the Suez canal and warrants further work. Within the Lessepsian clade, the presence of many haplotypes (14 for Dloop and 11 for COI), obtained from individuals collected in 1996, 2000, 
and 2008, indicates that either large numbers of individuals entered the Mediterranean, or more likely multiple invading episodes may have occurred since the first Mediterranean record of the species in 1953.

\section{Differential invading potential in cryptic species}

Obtaining reciprocally monophyletic clades that are not geographically separated using mitochondrial markers is not new (Horne et al. 2008). The explanation of these observations, however, is often tentative because only potential causes of breeding barriers can be evoked based on most likely scenarios. If these clades are the evidence of the early stages of speciation, then some predictions may be made. If interbreeding has stopped very recently, sorting may have occurred at the mitochondrial level, but may not be present for nuclear markers, for which sorting occurs 4 times slower (e.g. Palumbi et al. 2001). In contrast, if breeding barriers were established for a long enough time, sorting would occur for both mitochondrial and nuclear markers, resulting in reciprocally monophyletic clades in both types of markers, which is what is expected in bona fide species. In our case, we found that mitochondrial sequences partition into 2 well-defined clades, resulting from a lack of gene flow between them. In contrast, nuclear markers do not sort the same individuals, meaning that we sampled individuals at a time that is after mitochondrial sorting but before nuclear sorting. In addition, introgression may exhibit a different behavior in nuclear and mitochondrial markers, and nuclear markers may have introgressed more effectively than mitochondrial ones, accentuating the observed difference in nuclear and mitochondrial patterns. More importantly, the 2 mitochondrial clades have different characteristics. One mitochondrial clade includes exclusively Red Sea individuals, while the other clade includes both Red Sea and Mediterranean individuals. It is likely that invading opportunities occur frequently, as is shown by the large number of Mediterranean haplotypes. This suggests that individuals from the 2 clades have had opportunities to invade, but only one was able to effectively establish a population in the Mediterranean. It is possible that one clade invaded first, and was then able to ecologically exclude the invader that would have arrived next, due to demographic effects (Allee effect) or ecological competition (Taylor \& Hastings 2005). However, the fact that more individuals collected from Eilat are in the Red Sea clade than in the Lessepsian clade is puzzling. If this result is a reflection of the relative abundance of the 2 cryptic species, then the potential for invasion is not simply related to stochastic events. Yet, determining the relative abundance of the 2 clades is not easy. First, it is not easy to quantify individuals that are morphologically identical, and second, the staging area to cross the Suez Canal is more likely to be in the Suez area, a region that we did not sample. Access to sampling in that region is not easy, for safety and political reasons, and unfortunately our attempts to obtain samples from that region failed.

Samples from the Red Sea that were recovered in the 2 mitochondrial clades were collected sympatrically in the same beach seining set. After genotyping individuals, we went back to the voucher specimens and tried to find morphological differences between samples that belong to the 2 different clades. Specifically, the shape of the preopercular spines is a very variable feature in Callionymus and are commonly used for taxonomical purposes (Fricke 1983). In this case, preopercular spines did show variability in $C$. filamentosus, yet we did not find characteristic differences between individuals from the 2 mitochondrial clades. We therefore conclude that these 2 mitochondrial clades may represent the early stages of speciation in C. filamentosus, and the lack of apparent morphological differences indicates that we are in the presence of incipient cryptic species. The difference in invading potential in these 2 groups may be due to a number of factors. Potential for local adaptation is a tempting idea, yet phylogeographic differences may blur such a signal (Bierne et al. 2011). Stochastic events may overrule the system, although our data indicate that the clade that successfully invaded the Mediterranean seems to be the least abundant one. Finally, ecological characteristics of the 2 proposed cryptic species may be different, as was shown in other systems (Hebert et al. 2004, Smith et al. 2007), and favor invading potential in one and not the other.

The opening of the Suez Canal resulted in an ecological turmoil but also a grand experiment. Here, we have the unique opportunity to understand a facet of cryptic speciation that is usually very difficult to address, namely the ecological significance of genetic diversification. We propose that Lessepsian bioinvasions offer a very good model to study the balanced effects of ecology and selection on invading potential.

Acknowledgements. We thank H. van Pelt for information on Callionymus sequences. This project was partially funded by a grant from the University of California Santa Cruz to G.B. and a grant from the Department of Fishery, Ministry of Agriculture, Israel, to D.G. 


\section{LITERATURE CITED}

Azzurro E, Golani D, Bucciarelli G, Bernardi G (2006) Genetics of the early stages of invasion of the Lessepsian rabbitfish Siganus luridus. J Exp Mar Biol Ecol 333:190-201

Bariche M, Heemstra P (2012) First record of the blacktip grouper Epinephelus fasciatus (Teleostei: Serranidae) in the Mediterranean Sea. Mar Biodivers Rec Vol 5

Ben-Tuvia A (1953) New Erythrean fishes from the Mediterranean coast of Israel. Nature 172:464-465

Bernardi G, Golani D, Azzurro E (2010) The genetics of Lessepsian bioinvasions. In: Golani D, AppelbaumGolani B (eds) Fish invasions of the Mediterraneanchange and renewal. Pensoft, Sofia, p 71-84

Bierne N, Welch J, Loire E, Bonhomme F, David P (2011) The coupling hypothesis: why genome scans may fail to map local adaptation genes. Mol Ecol 20:2044-2072

Brothers EB, Thresher RE (1985) Pelagic duration, dispersal and the distribution of Indo-Pacific coral reef fishes. In: Reaka ML (ed) The ecology of coral reefs, 1st edn, Vol 3. National Oceanic and Atmospheric Administration, Washington, DC, p 53-69

Bucciarelli G, Golani D, Bernardi G (2002) Genetic cryptic species as biological invaders: the case of a Lessepsian fish migrant, the hardyhead silverside Atherinomorus lacunosus. J Exp Mar Biol Ecol 273:143-149

> Chow S, Hazama K (1998) Universal PCR primers for S7 ribosomal protein gene introns in fish. Mol Ecol 7: 1255-1256

Drummond AJ, Ashton B, Buxton S, Cheung M and others (2011) Geneious v5.4. www.geneious.com/

Excoffier L, Lischer HEL (2010) Arlequin suite ver 3.5: A new series of programs to perform population genetics analyses under Linux and Windows. Mol Ecol Resour 10: 564-567

Felsenstein J (1985) Confidence limits on phylogenies: an approach using the bootstrap. Evolution 39:783-791

Fricke R (1983) Revision of the Indo-Pacific genera and species of the dragonet family Callionymidae (Teleostei). Verlag von J. Cramer, Braunschweig

Geller JB, Darling JA, Carlton JT (2010) Genetic Perspectives on marine biological invasions. Ann Rev Mar Sci 2: 367-393

Golani D (2010) Colonization of the Mediterranean by Red Sea fishes via the Suez Canal-Lessepsian migration. In: Golani D, Appelbaum-Golani B (eds) Fish invasions of the Mediterranean-change and renewal. Pensoft, Sofia, p 145-188

Golani D, Orsi-Relini L, Massuti E, Quignard JP (2002) CIESM atlas of exotic species in the Mediterranean, Vol 1. In: Briand F (ed) Fishes. CIESM Publications, Monaco

Golani D, Azzurro E, Corsini-Foka M, Falautano M, Andaloro F, Bernardi G (2007) Genetic bottlenecks and successful biological invasions: the case of a recent Lessepsian migrant. Biol Lett 3:541-545

Hassan M, Bonhomme F (2005) No reduction in neutral variability of mitochondrial and nuclear genes for a Lessepsian migrant, Upeneus moluccensis. J Fish Biol 66: 865-870

Hassan M, Harmelin-Vivien M, Bonhomme F (2003) Lessep-

Editorial responsibility: Philippe Borsa,

Montpellier, France sian invasion without bottleneck: example of two rabbitfish species (Siganus rivulatus and Siganus luridus). J Exp Mar Biol Ecol 291:219-232

Hebert PDN, Penton EH, Burns JM, Janzen DH, Hallwachs W (2004) Ten species in one: DNA barcoding reveals cryptic species in the neotropical skipper butterfly Astraptes fulgerator. Proc Natl Acad Sci USA 101: 14812-14817

Horne JB, van Herwerden L, Choat JH, Robertson DR (2008) High population connectivity across the Indo-Pacific: congruent lack of phylogeographic structure in three reef fish congeners. Mol Phylogenet Evol 49:629-638

Katoh K, Misawa K, Kuma K, Miyata T (2002) MAFFT: a novel method for rapid multiple sequence alignment based on fast Fourier transform. Nucleic Acids Res 30: 3059-3066

Knowlton N (2000) Molecular genetic analyses of species boundaries in the sea. Hydrobiologia 420:73-90

Lee WJ, Conroy J, Howell WH, Kocher TD (1995) Structure and evolution of teleost mitochondrial control regions. J Mol Evol 41:54-66

> Librado P, Rozas J (2009) DNAsp v5: a software for comprehensive analysis of DNA polymorphism data. Bioinformatics 25:1451-1452

Palumbi SR, Cipriano F, Hare MP (2001) Predicting nuclear gene coalescence from mitochondrial data: the threetimes rule. Evolution 55:859-868

Pancucci-Papadopoulou AM, Zenetos A, Corsini-Foka M, Politou C (2005) Update of marine aliens in Hellenic waters. Mediterr Mar Sci 6:147-158

Sambrook J, Fritsch EF, Maniatis T (1989) Molecular cloning: a laboratory manual. Cold Spring Harbor Laboratory Press, Woodbury, NY

Sevilla RG, Diez A, Noren M, Mouchel O and others (2007) Primers and polymerase chain reaction conditions for DNA barcoding teleost fish based on the mitochondrial cytochrome $\mathrm{b}$ and nuclear rhodopsin genes. Mol Ecol Notes 7:730-734

Shimodaira H, Hasegawa M (1999) Multiple comparisons of log-likelihoods with applications to phylogenetic inference. Mol Biol Evol 16:1114-1116

Smith MA, Wood DM, Janzen DH, Hallwachs W, Hebert PDN (2007) DNA barcodes affirm that 16 species of apparently generalist tropical parasitoid flies (Diptera, Tachinidae) are not all generalists. Proc Natl Acad Sci USA 104:4967-4972

Swofford DL (2003) PAUP*. Phylogenetic analysis using parsimony ( ${ }^{*}$ and other methods). Version $4.0 \mathrm{~b} 10$. Sinauer Associates, Sunderland, MA

- Taylor CM, Hastings A (2005) Allee effects in biological invasions. Ecol Lett 8:895-908

Tortonese E (1953) Su alcuni pesci Indo-Pacifici immigrati nel Mediterraneo orientale. Boll Zool 20:73-81

Ward RD, Zemlak TS, Innes BH, Last PR, Hebert PDN (2005) DNA barcoding Australia's fish species. Philos Trans R Soc Lond B 360:1847-1857

Zwickl DJ (2006) Genetic algorithm approaches for the phylogenetic analysis of large biological sequence datasets under the maximum likelihood criterion. PhD dissertation, The University of Texas at Austin, TX

Submitted: May 13, 2011; Accepted: December 22, 2011

Proofs received from author(s): March 21, 2012 